\title{
Portable Electronic Device: An Alternative Tool for Surgical Magnification
}

Audrey Jose Miranda-Diaz, MD,2; Carlos Andrés Melo-Luna, MD, PhD; Raul Devia-Rodriguez, MD²;

John Henry Reina, MD, PhD; Maria Isabel Cadena-Rios, MD, MSC2,4*

'Universidad Santiago de Cali, Cali, Colombia

2Research Group for Plastic, Aesthetic, and Reconstructive Surgery C.E.R., Cali, Colombia

${ }^{3}$ Centre for Bioinformatics and Photonics (CIBioFi), Universidad del Valle, Cali, Colombia

${ }^{4}$ Department of Plastic Surgery, Universidad del Valle, Cali, Colombia

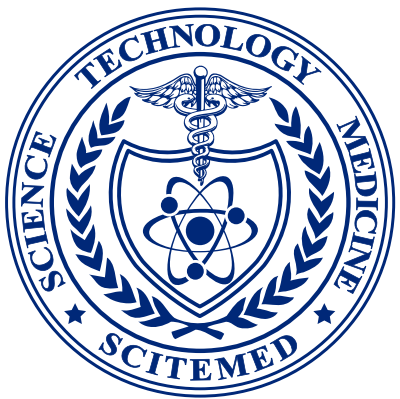

\begin{abstract}
Introduction: Portable electronic devices (PEDs) such as smartphones, laptops, and tablets have become important tools in the medical field for monitoring, learning, and clinical diagnosis. The link between PEDs gadgets and high definition quality cameras with zoom enhances, illumination, and video recording allows their use as magnification devices for microsurgery training.

Methods: To compare different portable electronic devices (PEDs) with the classical surgical magnification devices, we conducted two sets of experiments that evaluated the behavior of the field of view, magnification, and amplification at different working distances for the PEDs. In the evaluation of the image quality, four variables were characterized by PEDs, ending on each team at different working distances. Results: In the comparison between PEDs and loupes, it was found that there was superiority in terms of the amplification power of the PEDs; superiority was also observed for the field of view, except with PED 4 and PED 5, where there were no statistically significant differences.

Conclusion: PEDs can be used as alternative means of magnification in microsurgery considering that they are superior to surgical loupes regarding the field of view and magnification, with characteristics having a behavior closer to the surgical microscopy settings. Also, considering their characteristics, they can be cheaper than loupes with better performance, besides greater accessibility in the market and plasticity compared to the classical magnification devices.
\end{abstract}

\section{INTRODUCTION}

Microsurgery training is a compulsory requirement in most surgical residency programs around the world. The aim of this paper is based on the importance of microsurgery techniques as a fundamental tool in the development of complex procedures in diverse medical and surgical specialties. Moreover, microsurgery training requires, besides surgical skills, the development of dexterities regarding the use of magnification devices such as surgical loupes and microscope. These devices are expensive and frequently inaccessible tools. Additionally, lack of time, infrastructure, restricted access, and adequate equipment by the surgeons are important problems that must be addressed $[2,3]$.

Nowadays, mobile devices like smartphones, laptops, and tablets have impacted industries such as engineering, metrology, telecommunications, agriculture, and health sciences, becoming an important tool for monitoring, learning, and clinical diagnosis, among others in the medical field $[4,5]$. The link between mobile devices' gadgets and high definition cameras with zoom enhances, illumination, and video recording has allowed their uses as magnification tools for microsurgery training [6-9].

Additionally, portable electronic devices (PEDs) have been used in the surgical field for scanning and mapping applications for flaps through near-infrared spectroscopy for the evaluation of the rate of intraoperative permeability. This has been helpful to the clinical practice of surgeons using these devices.

We performed an exhaustive scientific literature review (from 01/8/2018 to $01 / 03 / 2019$ ) in the medical databases using PubMed, Medline, Science Direct, Scielo, Embase, and Google Scholar. Our search included six terms: Microsurgery Training, Competence-Based Education, Training, Smartphone, Technology, and Motor Skills. Within our review, we did not find any research comparing between PEDs and the classical magnification devices regarding magnifying characteristics, image quality, and costs.
Nevertheless, all the available researches describing specific training techniques or exercises for training in microsurgery using PEDs have lower level evidences (papers such as letters to the editors and unpublished observational studies). [2,3,10].

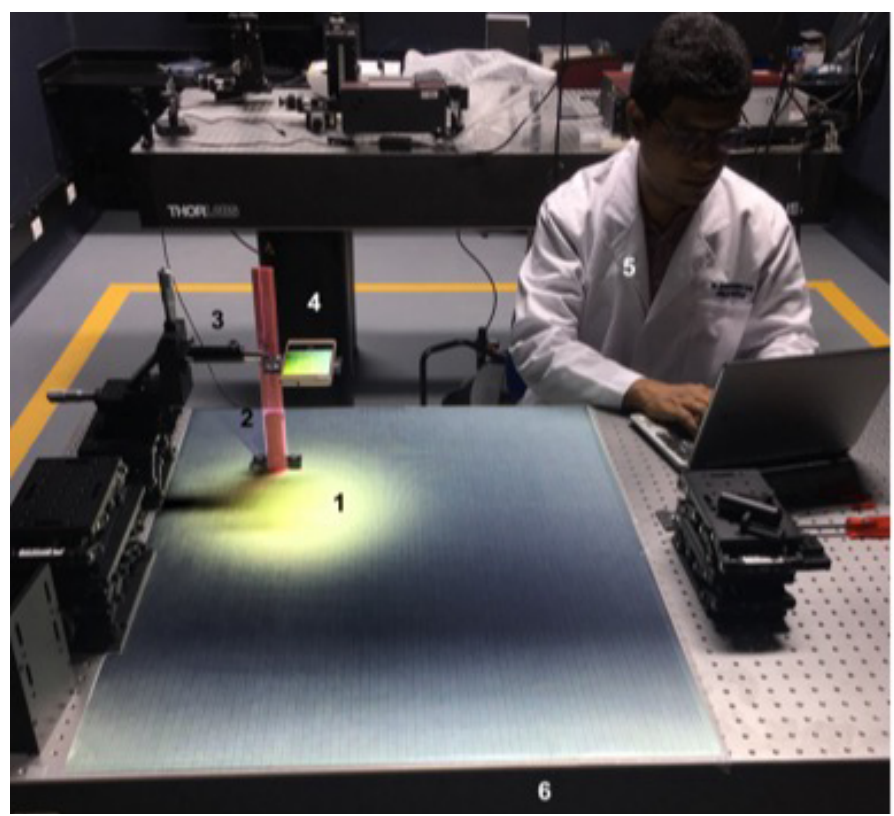

Figure 1. Experimental setup for portable electronic devices testing. The number 1 indicates millimetric sectioned pattern; the number 2 indicates working distance measure system; the number 3 indicates elevation lab jacks and micrometrical XYZ stage; the number 4 indicates portable electronic device holder; the number 5 indicates experimenter; and the number 6 indicates vibration isolating optical table. 
(A)

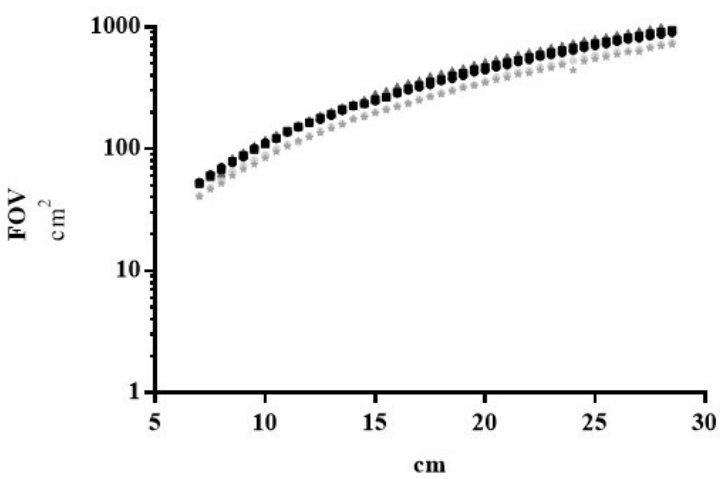

Working Distance
(B)

Field of view in DZ-M

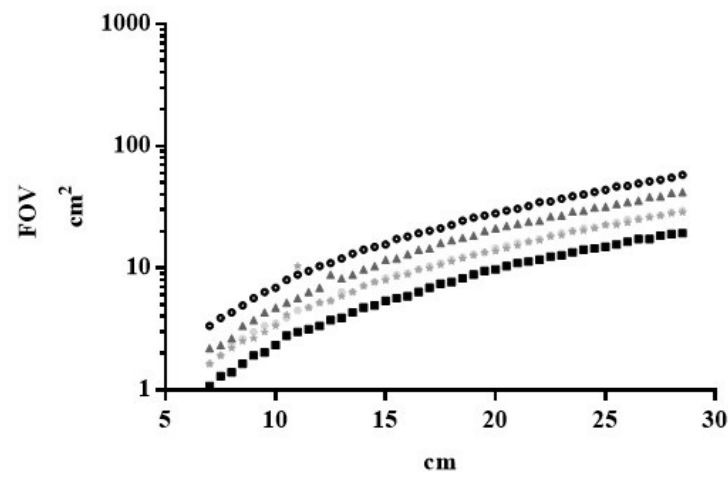

Working Distance

Figure 2. PEDs field of view and working distance. (A) Curve field of view in DZ-O. (B) Curve field of view in DZ-M. DZ-0, no digital zone; DZ-M, maximal digital zoom; FOV, field of view; PED, portable electronic device.

(A) Magnification in relation to screen size in DZ-0

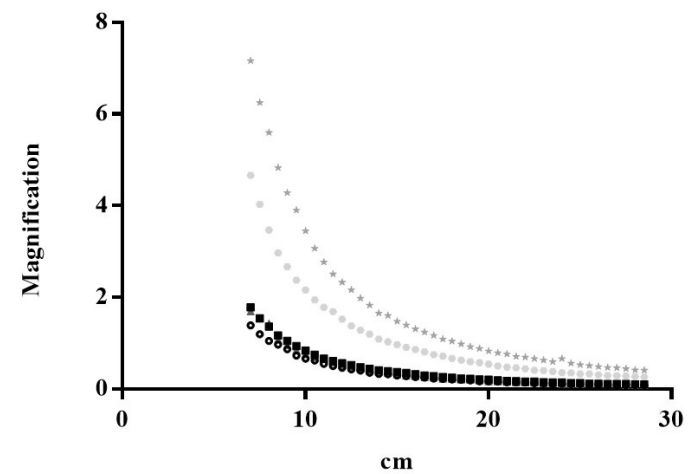

Working Distance
(B) Magnification in relation to screen size in DZ-M

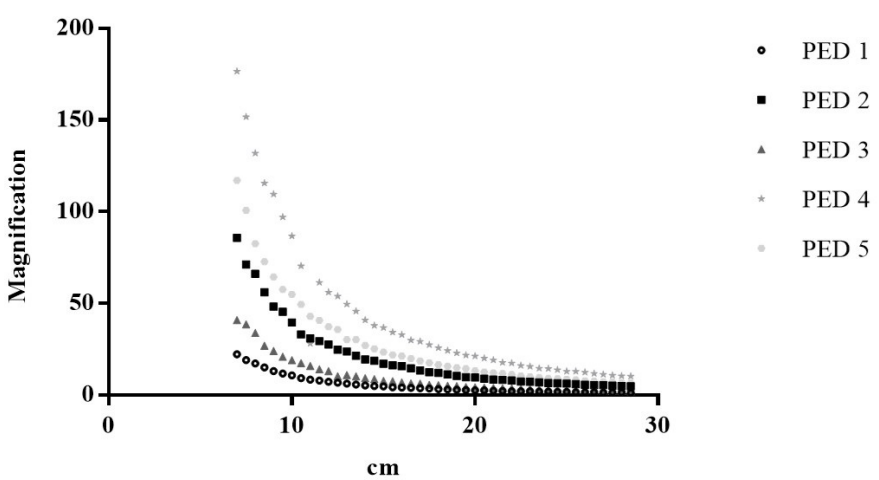

Working Distance

Figure 3. Image magnification (experimental) and working distance. (A) Curve in DZ-O. (B) Curve in DZ-M. DZ-0, no digital zone; DZ-M, maximal digital zoom; PED, portable electronic device.

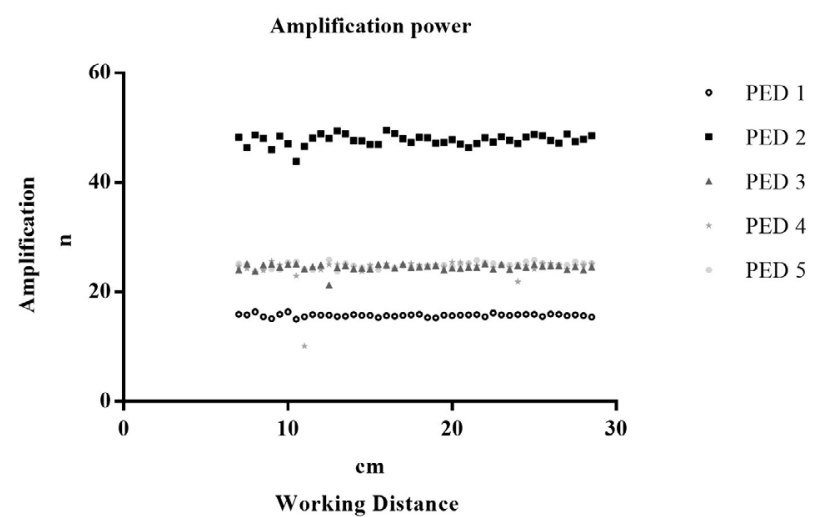

Figure 4. Amplification power and working distance. PED, portable electronic device.
This study aims to develop the first statistical comparison between alternative and conventional magnification devices focused on microsurgery training. Besides, it describes our experience in the use of a smartphone as an accessible, versatile, and affordable alternative magnification device for microsurgery training. Therefore, unanimated models have been used to develop surgical skills and dexterities in operating magnification devices as the bases for microsurgery training in all surgical medical residencies $[1,11]$.

\section{METHODS}

To compare the surgical classical magnification devices with the PEDs technologies, we experimentally evaluated the field of view (FOV), the magnification, and amplification for different working distances (WD) in the Multispectral Optics and Biophotonics Laboratory (LABIFOM), which belonged to the Research Centre for Bioinformatics and Photonics (CIBioFi). These tests allowed us to compare between the selected PEDs (PED 1, Huawei P9 Lite; PED 2, Google Pixel 2 XL; PED 3, iPhone XR; PED 4: iPad Air 9.7; PED 5, iPad mini 4.) characteristics through four technical parameters under controlled conditions of humidity $(50 \%)$, temperature $\left(22^{\circ} \mathrm{C}\right)$, and luminosity (low). 
(A) Field of view in DZ-0

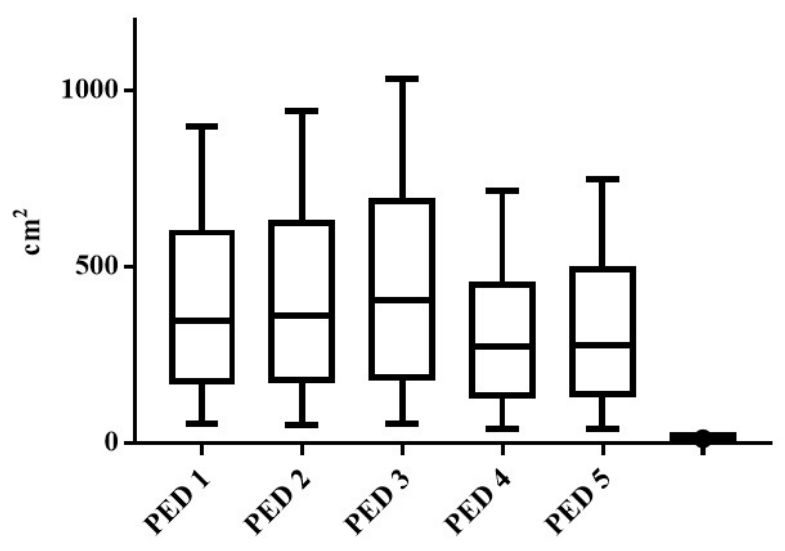

(C) Magnification in relation to screen size in $\mathrm{DZ}-0$

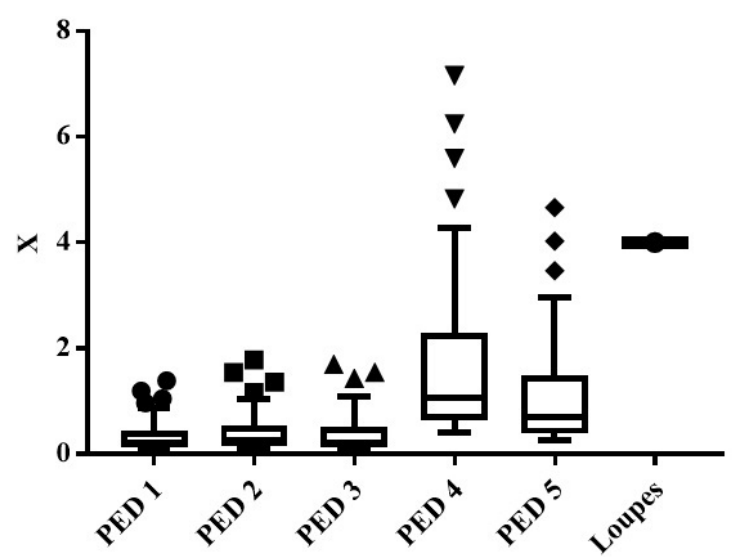

(B) Fiel of view in DZ-M

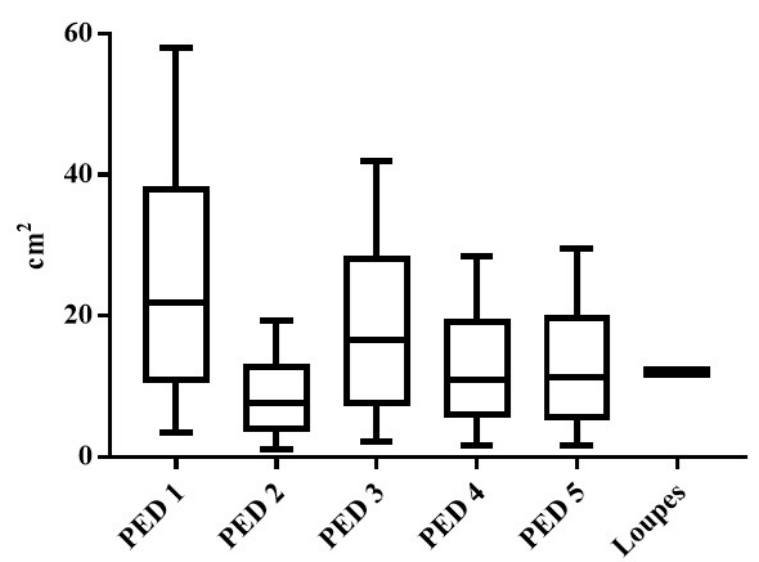

(D) Magnification in relation to screen size in DZ-M

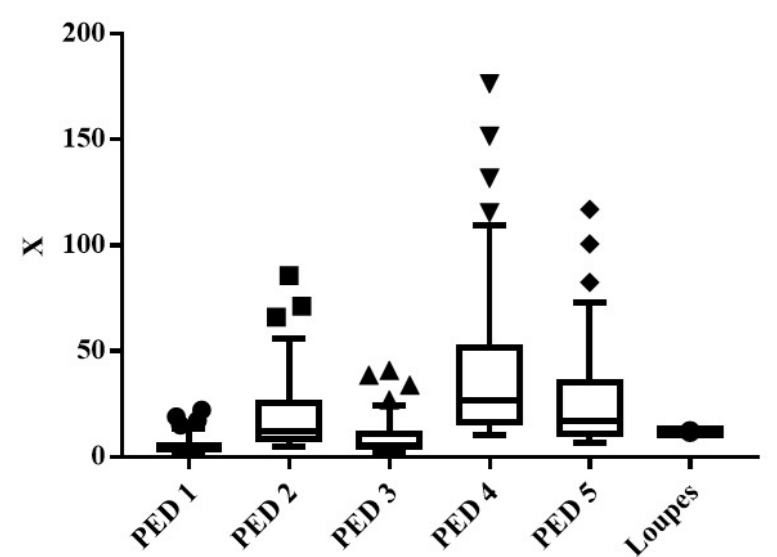

Figure 5. Box plot of field of view and magnification between PEDs and surgical loupes. (A and B). Statistical comparison of the PEDs to different FOV ( $\left(\mathrm{cm}^{2}\right)$. (C and D) Statistical comparison of the PEDs with respect to their image magnification. FOV, field of view; PED, portable electronic device.

Comparison of power amplification

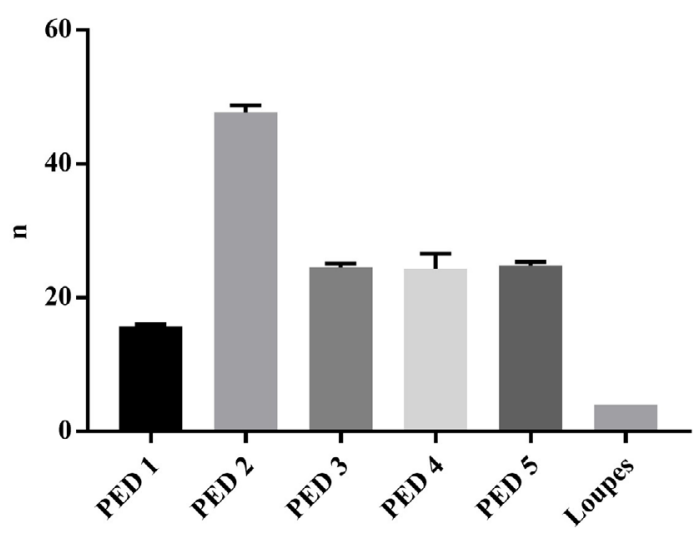

Figure 6. Amplification power comparison. Distribution of amplification power mean for the different PEDs and loupes. PED, portable electronic device.
First, we set up each PED in a displacement XYZ stage with a fine resolution of $25 \mu \mathrm{m}$ per loop to observe a millimetric sectioned pattern. The measurement system was attached to a vibration isolating table (Figure 1). Moreover, in order to diminish intra and inter-observational confounding, the experiments were performed by only one operator to whom the visual capability was a measure through an optometric examination to ensure standard conditions (Figure 1).

As a classical surgical device to be compared with, we employed the Galilean magnifying glasses with $4 X$ of magnification and $420 \mathrm{~mm}$ of working distance (Northope) under the same conditions as the PEDs. On the other hand, we also included the surgical microscope just to define a benchmark with the commercial specifications of working distance and magnification. The PEDs tested were labeled from 1 to 5 (PED 1-5) and the specs were displayed in Table 1.

\section{FOV Determination of PEDs at Different WD}

We captured 44 pictures at different WD by each PED (a total amount of 202 pictures) to compare the FOV without digital zoom (DZ-0) and with the maximal digital zoom possible (DZ-M). In this sense, we defined two kinds of FOV: an apparent field of view (AFOV) and another real field of view (RFOV). The 
ORIGINAL

\begin{tabular}{llllll}
\hline Table 1. PEDs specifications & \multicolumn{1}{l}{} \\
\hline & PED 1 & PED 2 & PED 3 & PED 4 & PED 5 \\
\hline Focal length & $27 \mathrm{~mm}$ & $27 \mathrm{~mm}$ & $28 \mathrm{~mm}$ & $31 \mathrm{~mm}$ & Not available \\
\hline Image resolution & $1080 \times 1920$ & $1440 \times 2880$ & $1792 \times 828$ & $2048 \times 1536$ & $1536 \times 2048$ \\
\hline Pixel density & 424 & 537 & 326 & 264 & 324 \\
\hline Sensor size & $1 / 3^{\prime \prime}\left(17.3 \mathrm{~mm}^{2}\right)$ & $1 / 2.55^{\prime \prime}\left(24.7 \mathrm{~mm}^{2}\right)$ & $1 / 2.55^{\prime \prime}\left(24.7 \mathrm{~mm}^{2}\right)$ & $1 / 3^{\prime \prime}\left(17.3 \mathrm{~mm}^{2}\right)$ & Not available \\
\hline Field number & $\mathrm{f} / 2.0$ & $\mathrm{f} / 1.8$ & $\mathrm{f} / 1.8$ & $\mathrm{f} / 2.4$ & Not available \\
\hline Screen size & $5.2^{\prime \prime}$ & $6^{\prime \prime}$ & $6.1^{\prime \prime}$ & $9.7^{\prime \prime}$ & $7.9^{\prime \prime}$ \\
\hline
\end{tabular}

PED, portable electronic device.

\begin{tabular}{|c|c|c|c|c|c|c|}
\hline & \multicolumn{6}{|c|}{ Working Distance $\left(\mathrm{cm}^{2}\right)$} \\
\hline & Loupes & PED 1 & PED 2 & PED 3 & PED 4 & PED 5 \\
\hline $\mathrm{RM}$ & $3.5 x$ & $15 x$ & $47 x$ & $24 x$ & $24 x$ & Not available \\
\hline DZ-0 & \multirow[t]{2}{*}{12} & 1946.91 & 2054.08 & Not available & 1567.51 & 1548 \\
\hline DZ-M & & 122.8 & 42.75 & 98.79 & 64.4 & 65.1 \\
\hline
\end{tabular}

Comparison between field of view of the PEDs and surgical loupes in DZ-0, and DZ-M at a working distance of $42 \mathrm{~cm}$. The area reported for the surgical loupes corresponds to the unique characteristics confirmed in the laboratory. DZ-0, no digital zone; DZ-M, maximal digital zoom; PED, portable electronic device; RM: Ratio of magnification.

\begin{tabular}{lllll}
\hline Table 3. Comparison of sharpness image in PEDs in DZ-M & & & \\
\hline PEDs & WD minimum $(\mathrm{cm})$ & WD maxima $(\mathrm{cm})$ & FOV minimum $\left(\mathrm{cm}^{2}\right)$ & FOV maxima $\left(\mathrm{cm}^{2}\right)$ \\
\hline PED 1 & 5 & 16.5 & 1.65 & 19.38 \\
\hline PED 2 & 7 & 13.5 & 1.08 & 4.32 \\
\hline PED 3 & 11.5 & 24.5 & 6.38 & 31.36 \\
\hline PED 4 & 7 & 15.5 & 1.65 & 8.5 \\
\hline PED 5 & 6.5 & 19.0 & 14 & 13.02 \\
\hline
\end{tabular}

DZ-M, maximal digital zoom; FOV: Field of view; PED, portable electronic device; WD, working distance.

Table 4. Comparison of $P$ values between field of view, amplification, and optical magnification of the PEDs

\begin{tabular}{|c|c|c|c|c|}
\hline \multirow[t]{2}{*}{ Comparisons } & \multicolumn{2}{|c|}{ Area visual } & \multirow[t]{2}{*}{ Amplification power } & \multirow[t]{2}{*}{ Ratio magnification } \\
\hline & DZ-0 & DZ-M & & \\
\hline PED 1 vs. PED 2 & $>0.9999$ & $<0.0001$ & $<0.0001$ & $<0.0001$ \\
\hline PED 1 vs. PED 3 & $>0.9999$ & 0.8913 & $<0.0001$ & $<0.0001$ \\
\hline PED 1 vs. PED 4 & $>0.9999$ & 0.0033 & $<0.0001$ & $<0.0001$ \\
\hline PED 1 vs. PED 5 & $>0.9999$ & 0.0042 & $<0.0001$ & ND \\
\hline PED 2 vs. PED 3 & $>0.9999$ & 0.0008 & $<0.0001$ & $<0.0001$ \\
\hline PED 2 vs. PED 4 & 0.9317 & 0.3947 & $<00001$ & $<0.0001$ \\
\hline PED 2 vs. PED 5 & $>0.9999$ & 0.3412 & $<0.0001$ & ND \\
\hline PED 3 vs. PED 4 & 0.3100 & 0.5908 & $>0.9999$ & $>0.9999$ \\
\hline PED 3 vs. PED 5 & 0.5506 & 0.6753 & $>0.9999$ & ND \\
\hline PED 4 vs. PED 5 & $>0.9999$ & $>0.9999$ & $>0.9999$ & ND \\
\hline PED 1 vs. Loupes & $<0.0001$ & $<0.0001$ & $<0.0001$ & $<0.0001$ \\
\hline PED 2 vs. Loupes & $<0.0001$ & 0.0003 & $<0.0001$ & $<0.0001$ \\
\hline PED 3 vs. Loupes & $<0.0001$ & 0.0049 & $<0.0001$ & $<0.0001$ \\
\hline PED 4 vs. Loupes & $<0.0001$ & 0.7131 & $<0.0001$ & $<0.0001$ \\
\hline PED 5 vs. Loupes & $<0.0001$ & 0.6584 & $<0.0001$ & ND \\
\hline
\end{tabular}

DZ-0, no digital zone; DZ-M, maximal digital zoom; ND, do not determine; PED, portable electronic device. 


\begin{tabular}{lllll}
\hline \multicolumn{2}{l}{ Table 5. Comparison between PEDs and magnification devices } & & & \\
\hline Dispositive & Magnification & WD $(\mathrm{mm})$ & FOV $\left(\mathrm{mm}^{2}\right)$ & Market price (USD) \\
\hline Microscope standard & $10-40 \mathrm{x}$ & $200-625$ & Not available & $3,469-129,122$ \\
\hline Surgical loupes & $3-8 x^{*}$ & $330-550^{*}$ & 120 & $159-923$ \\
\hline PED 1 & $15 x$ & $50-165$ & $16.5-193.8$ & 200 \\
\hline PED 2 & $47 x$ & $70-135$ & $10.8-43.2$ & 339 \\
\hline PED 3 & $24 x$ & $115-245$ & $63.8-313.6$ & 479 \\
\hline PED 4 & $24 x$ & $70-155$ & $16.5-85$ & 329 \\
\hline PED 5 & Not available & $65-190$ & $14-130.2$ & 399 \\
\hline
\end{tabular}

*Depending type loupes. FOV: Field of view; PED, portable electronic device; PED 1, Huawei P9 Lite; PED 2, Google Pixel 2 XL; PED 3, iPhone XR; PED 4: iPad Air 9.7; PED 5, iPad mini 4; WD, working distance.

AFOV is defined as the physical size of the PED display area (Table 1) and the RFOV is determined with the millimetric pattern in the taken picture. Those parameters (RFOV at DZ-0 and DZ-M) were measured from 7.0 to $28.5 \mathrm{~cm}$ at variations of $0.5 \mathrm{~cm}$ (Figure 2).

\section{PEDs Magnification (M) and Working Distances}

We defined the magnification as the ratio between the AFOV and the RFOV, $=\frac{A F O V}{R F O V}$, and the amplification power as $n=\frac{R F O V_{A D-0}}{R F O V_{A D-M}}$, which described the number of times in which an image was zoomed. The obtained data were digitalized employing Microsoft Excel 8.0 and exported to GraphPad Prism versión 7.0 to perform the statistical analysis.

\section{PEDs Magnification Quality Regarding Cameras Sensor Size}

To assess the quality of the images offered by the different PEDs, we considered technical parameters such as the focal distance $(f)$, the display resolution, the number of pixels per inch (ppi), and the sensor size (Table 1). Additionally, the magnification (magnification ratio) was calculated in relation to the sensor size in DZ-0 and DZ-M for each equipment with the following formula, $m=\frac{\text { Sensor size }\left(\mathrm{mm}^{2}\right)}{R F O V\left(\mathrm{~mm}^{2}\right)}$

Then, the WD ranges (maximum and minimal) for the PEDs were determined by the visualization with the appropriate resolution of a 9.0 surgical needle (FOV $=5 \mathrm{~mm} \times 5 \mathrm{~mm}$ ). In addition, we calculated the ratio between the magnifications in relation to the size of the sensor in DZ-0 and DZ-M.

\section{RESULTS}

\section{PEDs Field of View and Focus Distance}

In the relation of the FOV of the PEDs in DZ-0, similar progression curves were evident for all the teams, however, this relationship in DZ-M showed different ranges, where the PED 1 showed a curve of greater observation of the FOV $\left(3.36 \mathrm{~cm}^{2}-58.08 \mathrm{~cm}^{2}\right)$ and PED 2 the smallest $\left(1.08 \mathrm{~cm}^{2}-19.38 \mathrm{~cm}^{2}\right)$ (Figure 2).

\section{Magnification vs. working distance}

Figure 3 shows a negative correlation between magnification and working distance: as working distance increases, there is lower magnification. Here, the curves PED 4 (DZ-0: 0.41 - 7.19 / DZ-M: 10.22 - 176.6) and PED 5 (DZ-0: 0.26 - 4.66 / DZ-M: 6.53 - 117.2) show the best magnification. No value was found where a point of convergence occurred between all the PEDs in the magnification curves in DZ-0 and DZ-M.

\section{Amplification power and working distance}

The amplification power for each PED showed a constant behavior at each WD, whereas PED 2 ( $\underline{x}=15.7)$ presented a greater amplification power and
PED 1 ( $\underline{x}=47.6$ ) the lowest (Figure 4). The magnification ratio showed values similar to the amplification power except for PED 5. PED 5 could not be determined as there was no reference to the size of the equipment sensor (Table 1).

\section{Image quality}

The PEDs with screen resolution, pixel density, the highest sensor size and the smaller f-number showed the best image quality (Table 1). It should be taken into account that to observe a 9.0 needle, the minimum sensor resolution was $750 \times 750$ pixels; this value was obtained by calculating the minimum FOV required to observe the object.

$$
\text { Sensor resolution }=\text { Image resolution }=2 \cdot\left(\frac{F O V}{\text { Smallest detail }}\right)
$$

\section{Smartphones and surgical loupes}

When comparing the field of view observed at the working distance of standard surgical loupes with the PEDs, greater field of views were evidenced in the equipment than in the loupes, either in DZ-0 or in DZ-M. The FOV in PED 3 in DZ-0 could not be obtained due to alterations of the secondary image as a result of the optical effects of the device (Table 2).

\section{Working distance with adequate resolution of the PEDs in DZ-M and microsurgery}

A comparative table was made with the maximum and minimum values of WD with sufficient resolution to observe a 9.0 needle and its relationship with the FOV; where, PED 1 showed the smallest viewing distance $(5 \mathrm{~cm})$ and PED 4 the largest $(24.5 \mathrm{~cm})$; and in the case of the FOV, PED 2 showed the smallest $\left(1.8 \mathrm{~cm}^{2}\right)$ and PED 3 the greater $\left(31.36 \mathrm{~cm}^{2}\right)$ (Table 3$)$.

The surgical loupes have a fixed lens and magnification system designed to have a correct display at a single working distance that strictly conforms to the operator's display, which is not included in the comparison in Table 3.

\section{Statistical Analysis}

A non-parametric multivariate analysis of a randomized block experimental design was performed, running a Kruskal-Wallis test to statistically compare FOVs, amplification power, and magnification ratio between PEDs. On the other hand, the comparison of the PEDs and the standard surgical loupes was carried out with a Wilcoson signed Rank Test taking theoretical comparison values for the loupes as a distance of $42 \mathrm{~cm}$, a magnification of $4 X$, and a FOV of $12 \mathrm{~cm}^{2}$.

Table 4 shows a statistically significant difference and superiority in FOV of PED 1 with respect to PED 2, 4 and 5 in DZ-M. When comparing the amplification power and the magnification ratio, it was found that PED 1 had a lower magnification power with respect to all other equipment with a statistically significant difference and PED 3, 4 and 5 showed no statistically significant differences in their magnification power between them.

In the comparison between PEDs and loupes, it was found that there was 
superiority in terms of the amplification power of the PEDs; superiority was also observed for FOV, except with PED 4 and PED 5 where there were no statistically significant differences (Figure 5 and Figure 6).

\section{DISCUSSION}

The use of PEDs as an alternative means of magnification for microsurgery training has been documented in the literature, but always from a subjective point of view and dependent on operator. This work aims to achieve a comparative analysis with objective terms and variables from the optical physics between the different PEDs and the classical magnification devices, and subsequently its usefulness in microsurgery training. It should be mentioned that this work is the first of its kind.

For this work, the magnification was determined in relation to the screen size and the size of the sensor. The first one offers a quantification from what the surgeon observes, that is, the screen; but its utility will depend on how far the image has a good quality; whereas, in the second one since the size of the sensor is a value given by the manufacturer, the magnification calculation can have a greater degree of certainty. To compare the magnifications of the traditional devices and the PEDs, the magnification ratio and the amplification power were considering that the experimental and theoretical data of these last items were equivalent.

Therefore, it was found that PEDs had superiority over loupes in magnification, FOV, and versatility for modifying the working distances, allowing the surgeon greater mobility and freedom of their eyes and body movements, reducing the stress. Additionally, the PEDs play an important role in avoiding the poor posture one develops with the use of loupes in surgery. Besides, the PEDs have greater magnification with respect to classical magnification devices and their behavior is closer to the microscopes considering the variables compared and which allows modifying the WD (Table 5).

The objective of this article is for training purposes within the microsurgical technique. However, we hope that with further advancements in this field including the design of applications for image optimization, ergonomic support devices, and validation of PEDs as a surgical instrument, these devices can be used in the near future in clinical settings.

\section{CONCLUSION}

PEDs can be used as alternative means of magnification in microsurgery training considering that they are superior to surgical loupes in magnification, FOV and WD ranges, allowing greater operational versatility in microsurgical maneuvers, its behavior being closer to that of surgical microscopes in some optical characteristics. Additionally, these devices have a lower cost than microscopes and some brands of surgical loupes, greater accessibility in the market and innovation plasticity through technological and physical applications and accessories with respect to classical magnification devices. Although PEDs own advanced technological features such as high-quality cameras and electronic loupes applications to improve the visualizations, it is important to continue the development of better technological applications and accessories for microsurgical practice, and additionally, it is important to produce evidence of its application at surgery room.

\section{ARTICLE INFORMATION}

*Correspondence: Maria Isabel Cadena-Rios, MD, MSC. Department of Plastic Surgery, Universidad del Valle, Cali, Colombia. Research Group in Plastic, Aesthetic and Reconstructive Surgery C.E.R. Calle 5B5 \# 38-04, Cali, Colombia.

\section{E-mail: doctora@mariaisabelcadena.com}

Received: Oct. 16, 2019; Accepted:Jan. 13, 2020; Published: Jun. 18, 2020

\section{DOI: $10.24983 /$ scitemed.imj.2020.00130}

Ethics Approval and Consent to Participate: The study is in accordance with the ethical standards of the 1964 Helsinki declaration and its later amendments or comparable ethical standards.

Funding: The study was partially sponsored by grants from Gobernación del Valle del Cauca (Grant BPIN 2013000100007).

Conflict of Interest: The authors report no financial or other conflict of interest relevant to this article, which is the intellectual property of the authors.

Copyright (c) 2020 The Authors. This is an open-access article distributed under the terms of the Creative Commons Attribution 4.0 International License (CC-BY)

\section{ACKNOWLEDGMENT}

The authors would like to thank Dr. Angélica Castellanos for her work on the images, and thank the Colombian Science, Technology and Innovation Fund-General Royalties System (Fondo CTel-Sistema General de Regalías) and Gobernación del Valle del Cauca (Grant BPIN 2013000100007).

\section{REFERENCES}

1. Cadena-Rios MI, Rodas-Cortes Y. Magnification devices. Microsurgery experimental surgery training, Cali,Colombia: Universidad del Valle. 2018.

2. Kim DM, Kang JW, Kim JK, Youn I, Park JW. Microsurgery training using a smartphone. Microsurgery 2015;35(6):500-501.

3. Karakawa R, Yoshimatsu H, Nakatsukasa S, lida T. A new method for microsurgery training using a smartphone and a laptop computer. Microsurgery 2018;38(1):124-125.

4. Amiri IS, Azzuhri SRB, Jalil MA, et al. Introduction to photonics: principles and the most recent applications of microstructures. Micromachines ( $\mathrm{Ba}$ sel) 2018:11;9(9).

5. Yan Z, Hu T, Guo W, Deng A, Di J. A label-free immunosensor for determination of salbutamol based on localized surface plasmon resonance biosensing. Bioprocess Biosyst Eng 2014;37(4):651-657.

6. Pafitanis G, Hadjiandreou M, Miller R, et al. The use of mobile computing devices in microsurgery. Arch Plast Surg 2019;46(2):102-107.

7. Patel N, Rozen WM, Marsh D, et al. Modern use of smartphone applications in the perioperative management in microsurgical breast reconstruction. Gland Surg 2016;5(2):150-157.

8. Pongnumkul S, Chaovalit P, Surasvadi N. Applications of smartphone-based sensors in agriculture: a systematic review of research. Journal of Sensors 2015;18.

9. Huotarinen A, Niemelä M, Jahromi BR. Easy, efficient, and mobile way to train microsurgical skills during busy life of neurosurgical residency in resource-challenged environment. World Neurosurg 2017;107:358-361.

10. Choque-Velasquez J, Colasanti R, Collan J, Kinnunen R, Rezai Jahromi B, Hernesniemi J. Virtual reality glasses and "eye-hands blind technique" for microsurgical training in neurosurgery. World Neurosurg 2018;112:126130.

11. Velásquez, M. Microsurgery manual: microsurgical teaching techniques. Cali: Hospital Universitario del Valle, 1983. 